University of Wollongong

Research Online

Faculty of Engineering and Information

Faculty of Engineering and Information

Sciences - Papers: Part A

Sciences

$1-1-2014$

TRUS-probe integrated MOSkin detectors for rectal wall in vivo dosimetry in HDR brachytherapy: in phantom feasibility study

C Tenconi

Universita degli Studi di Milano-Bicocca

M Carrara

Fondazione Irccs Istituto Nazionale Tumori

M Borroni

Fondazione Irccs Istituto Nazionale Tumori

A Cerrotta

Fondazione Irccs Istituto Nazionale Tumori

D Cutajar

University of Wollongong, deanc@uow.edu.au

See next page for additional authors

Follow this and additional works at: https://ro.uow.edu.au/eispapers

Part of the Engineering Commons, and the Science and Technology Studies Commons

Research Online is the open access institutional repository for the University of Wollongong. For further information contact the UOW Library: research-pubs@uow.edu.au 


\title{
TRUS-probe integrated MOSkin detectors for rectal wall in vivo dosimetry in HDR brachytherapy: in phantom feasibility study
}

\author{
Abstract \\ The increasing complexity and high amount of dose per fraction delivered in prostate high dose rate \\ (HDR) brachytherapy treatments call for the implementation of accurate and effective methods for the \\ systematic and independent quality control of the overall treatment procedure. In this study, MOSkin \\ detectors were placed on a trans-rectal ultrasound (TRUS) probe with the aim of performing both imaging \\ and real time rectal wall in vivo dosimetry with the use of just one single instrument. After an adequate \\ calibration of the detectors, which was carried out in a solid water phantom, the use of MOSkins \\ integrated to the TRUS probe was studied in a gel phantom with a typical (simplified) prostate implant. \\ Measured and calculated doses from the treatment planning system were compared, with a resulting very \\ low average discrepancy of $-0.6 \pm 2.6 \%$. The results are very promising and of particular clinical \\ importance, however, further in vivo investigation is planned to validate the proposed method.

\section{Disciplines} \\ Engineering | Science and Technology Studies

\section{Publication Details} \\ Tenconi, C., Carrara, M., Borroni, M., Cerrotta, A., Cutajar, D., Petasecca, M., Lerch, M., Bucci, J., Gambarini, \\ G., Pignoli, E. \& Rosenfeld, A. (2014). TRUS-probe integrated MOSkin detectors for rectal wall in vivo \\ dosimetry in HDR brachytherapy: in phantom feasibility study. Radiation Measurements, 71 379-383.

\section{Authors} \\ C Tenconi, M Carrara, M Borroni, A Cerrotta, D Cutajar, M Petasecca, M Lerch, J Bucci, G Gambarini, E \\ Pignoli, and A Rosenfeld
}


TRUS-probe integrated MOSkin detectors for rectal wall in vivo dosimetry in HDR brachytherapy: In phantom feasibility study

C. Tenconi a,b, M. Carrara a, ${ }^{a,}$, M. Borroni a , A. Cerrotta c , D. Cutajar ${ }^{\text {, }}$, M. Petasecca ${ }^{\text {, }}$, M. Lerch ${ }^{d}$, J. Bucci e, G. Gambarini ${ }^{b}$, E. Pignoli ${ }^{a}$, A. Rosenfeld ${ }^{d}$

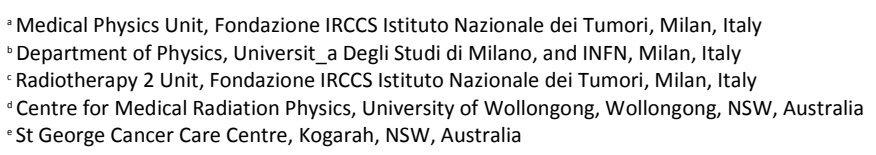

\section{ABSTRACT}

The increasing complexity and high amount of dose per fraction delivered in prostate high dose rate (HDR) brachytherapy treatments call for the implementation of accurate and effective methods for the systematic and independent quality control of the overall treatment procedure. In this study, MOSkin detectors were placed on a trans-rectal ultrasound (TRUS) probe with the aim of performing both imaging and real time rectal wall in vivo dosimetry with the use of just one single instrument. After an adequate calibration of the detectors, which was carried out in a solid water phantom, the use of MOSkins integrated to the TRUS probe was studied in a gel phantom with a typical (simplified) prostate implant. Measured and calculated doses from the treatment planning system were compared, with a resulting very low average discrepancy of $0.6 \pm 2.6 \%$. The results are very promising and of particular clinical importance, however, further in vivo investigation is planned to validate the proposed method.

\section{Introduction}

Brachytherapy (BT) delivers an extremely conformed high dose to the prostate, using either a low dose rate or a high dose rate (HDR) technique (Morton and Hoskin, 2013). With HDR, a single high activity source of short-range radiation (e.g., Ir-192) is remotely moved to predefined positions via temporarily needles that are implanted within the target. By adjusting the source dwell times in each one of the planned dwell positions, a tailored and customized dose distribution can be achieved to ensure optimal coverage of the defined treatment volume.

Nevertheless, HDR BT is a meticulous technique and procedural variations caused by factors such as patient motion, needle movements, anatomical changes occurring between imaging and treatments, as well as potential human inaccuracies or errors, may lead to a significant degradation of the plan (Thomadsen et al., 2003). Therefore, to ensure the delivered dose agrees within acceptable limits to that planned, rigorous quality assurance (QA) procedures should be implemented for independent treatment verification. In particular, effective and accurate QA methods need to be developed, as the complexity of BT delivery has rapidly increased (Palmer et al., 2012; Tanderup et al., 2013) and new highly fractionation schemes (i.e., 26-27Gy in two fraction or 19-21Gy in one single fraction) have recently been introduced (Morton and Hoskin, 2013).

In vivo dosimetry represents a useful QA tool, not only for avoiding treatment misadministration, but also for evaluating doses to organs at risk (i.e., urethra, rectum and bladder). Moreover, if performed on-line, it allows the constant monitoring of the dose delivered during the treatment, enabling the immediate intervention of the clinician if adverse dose readings are detected (Lambert et al., 2007).

A number of different systems to perform in vivo dosimetry have been developed, including passive integrating thermoluminescent dosimeters (TLDs) and active methods based on diodes, diamond detectors, optical fiber-coupled scintillators and MOSFETs (Fagerstrom et al., 2008; Rustgi, 1998; Toye et al., 2009; Waldhäusl et al., 2005; Zilio et al., 2006). Some of these have already been used to perform in vivo prostate dosimetry by directly placing them in either a needle in the prostate, a 
catheter in the urethra or in the rectum (Seymour et al., 2011; Suchowerska et al., 2011). As the trans-rectal ultrasound (TRUS) probe is one possible means of performing real time prostate HDR BT treatment planning, it would be advantageous to include dosimeters to the probe so that the probe itself would represent a tool for both imaging and in vivo rectal wall dosimetry. This system would in fact be particularly convenient, avoiding the insertion of extra invasive catheters or needles for the positioning of the detectors. In this study, a particular type of MOSFET dosimeters, MOSkins, were integrated to the TRUS probe, with phantom measurements of prostate brachytherapy implants performed.

\section{Materials and Methods}

\subsection{MOSkin dosimeters and the brachytherapy facility}

MOSkin detectors are particular type of MOSFET devices recently developed by the Centre for Medical Radiation Physics (CMRP), University of Wollongong, Australia. They are characterized by their novel packaging technology used to seal the sensor chip, which differentiates them from other commercial MOSFETs and makes them more suitable for dose measurements in steep dose gradients and for skin dosimetry. Their sensitive volume, defined by the volume of the gate oxide, is $4.8 \times 10^{-6} \mathrm{~mm}^{3}$. More specific information about MOSkin dosimeters may be found elsewhere (Qi et al., 2007; Kwan et al., 2009).

All the measurements in this study were carried out with a microSelectron-HDR remote afterloader device (Nucletron, Veenendaal, The Netherlands) provided with an Ir-192 source with active length of $3.6 \mathrm{~mm}$ and diameter of $0.65 \mathrm{~mm}$. The source is contained in a stainless steel capsule, laser welded to a steel cable for its remote positioning at required locations into the treatment catheters/needles.

\subsection{MOSkin calibration with the Ir-192 brachytherapy source}

MOSkin detectors calibrations were performed in a water-equivalent phantom constructed with several solid water slabs of various thickness placed in a stacked configuration to provide adequate backscatter conditions. One MOSkin at a time was positioned at the center of the phantom and was oriented with its sensitive volume in source direction.

A plastic needle for source positioning was inserted in the phantom with its centre at a distance of $21 \pm 0.1 \mathrm{~mm}$ from the MOSkin detector. To irradiate the detector, a single source dwell position was chosen right above the MOSkin (i.e., nearest point of the source catheter to the dosimeter) (Fig.1). In order to ensure that the response uncertainty associated with the MOSkin reader $( \pm 1 \mathrm{mV})$ was less than $1 \%$ of the threshold voltage change, a dwell time of $78 \mathrm{~s}$ was chosen. Each detector was irradiated three times and the corresponding voltage threshold measurements Vi were recorded and averaged to Vmean. The delivered dose at the calibration position Dcal was then calculated by a means of a commercial BT treatment planning system (TPS) (Oncentra Prostate, Nucletron, The Netherlands) which complies with the American Association of Physicists in Medicine (AAPM) TG-43 dose calculation formalism (Rivard et al., 2004). The calibration factor, N, was determined as $\mathrm{N}=$ Dcal/Vmean, given in $\mathrm{cGy} / \mathrm{mV}$. 


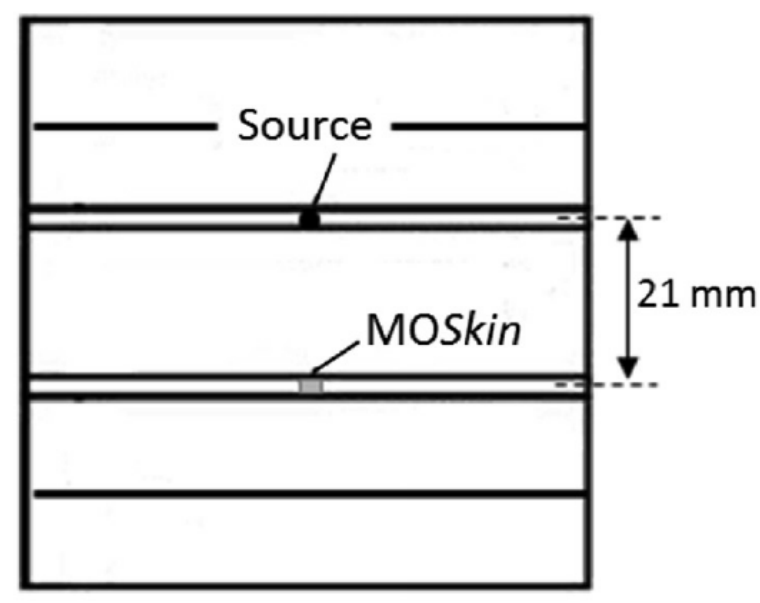

A)

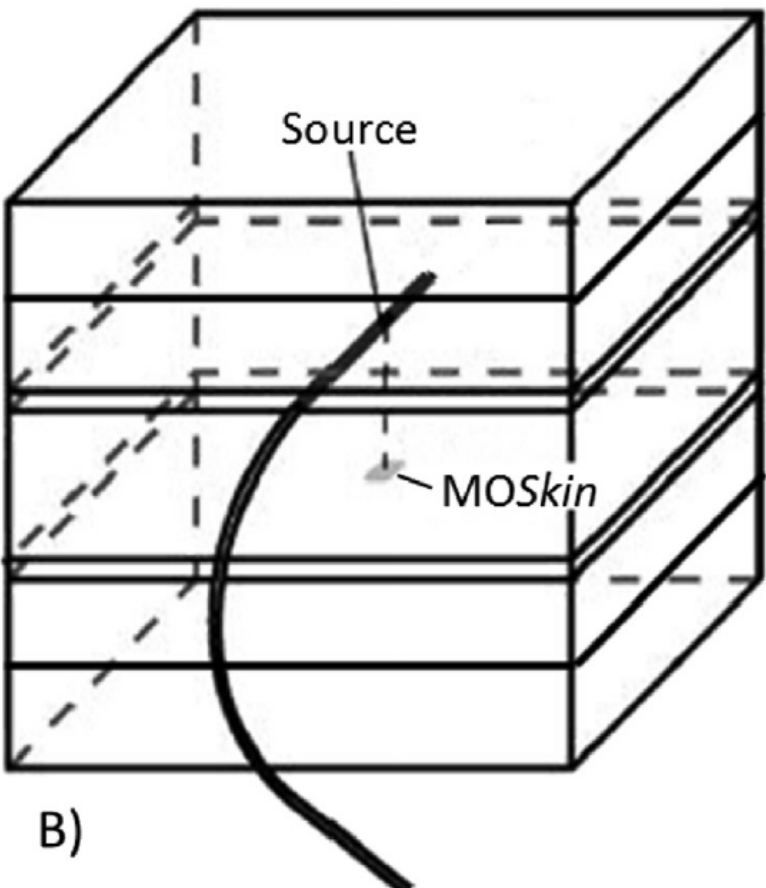

B)

Fig 1. Schematics of the experimental setup. A) The MOSkin detector was placed in the centre of the water-equivalent phantom. The orthogonal distance to the source catheter was of $21 \mathrm{~mm}$. B) During irradiation, the single dwell position of the $1 \mathrm{r}-192 \mathrm{source}$ was chosen in correspondence to the closest distance to the MOSkin detector (i.e. $21 \mathrm{~mm}$ ).

\subsection{Transrectal ultrasound-based prostate HDR brachytherapy}

Prostate ultrasound imaging by means of a TRUS probe is one of the possible image guidance modalities used to both guide the insertion of needles through the perineum and plan HDR BT treatments. Its use instead of CT imaging is constantly increasing (Batchelar et al., 2013). It would therefore be particularly convenient and effective to attach accurate dosimeters to the TRUS-probe in order to perform both imaging and real time rectal wall in vivo dosimetry by the use of just one single instrument.

\section{axial transducer}

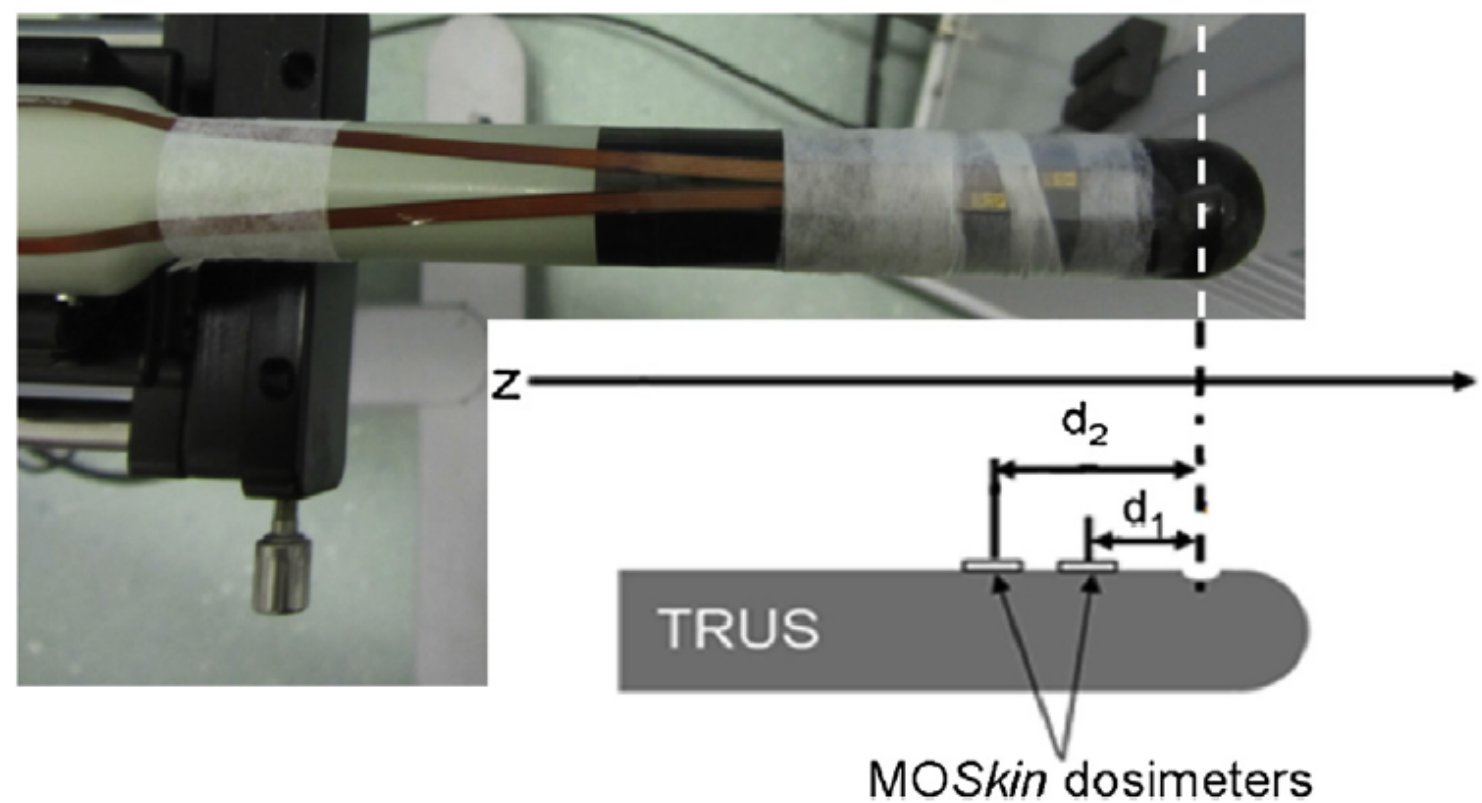

Fig 2. MOSkin dosimeters, positioned along the longitudinal axis of the probe, at a distance of $25 \mathrm{~mm}$ and $35 \mathrm{~mm}$ from the axial transducer, respectively. 
In this study, two MOSkin detectors were integrated to a TRUS probe which is commonly used in clinical practice. MOSkin detectors were positioned along the longitudinal axis, with an additional $2 \mathrm{~mm}$ silicon spacer between them and the surface of the probe. The sensitive surface of each detector was facing the target, with positions of $\mathrm{d} 1=25 \mathrm{~mm}$ and $\mathrm{d} 2=35 \mathrm{~mm}$ from the transversal transducer, respectively (Fig. 2).

\subsection{In phantom dosimetry of prostate brachytherapy treatments}

With the aim of performing dose measurements to the rectal wall with MOSkins integrated to the TRUS probe, a suitable gel phantom was created. The phantom has a cylindrical shape with length of $14.5 \mathrm{~cm}$, diameter of $17.6 \mathrm{~cm}$ and an off-center cavity simulating the rectum. Six interstitial needles were placed through a template inside the gel phantom over the cavity to reproduce a treatment implant (Fig. 3). Through the cavity, US-images were acquired in order to precisely localize needles and plan a treatment. A virtual prostate was created on the US images around the needles implant over a total longitudinal length of $25 \mathrm{~mm}$. Dose to the virtual prostate was optimized and the expected doses to each dosimeter evaluated. To identify the radial location of the detectors, the probe-image interface was taken as reference (Fig. 4) whereas the positions of the detectors along the longitudinal axis were identified at distances $\mathrm{d} 1$ and $\mathrm{d} 2$ from the base plane.

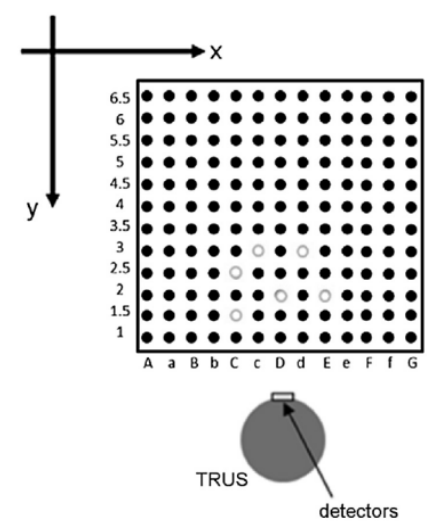

Fig 3. Schematic representation of the MOSkin detectors integrated on the TRUSprobe and of the six needles placed through the template into the gelatin phantom tosimulate a typical (simplified) clinical distribution.

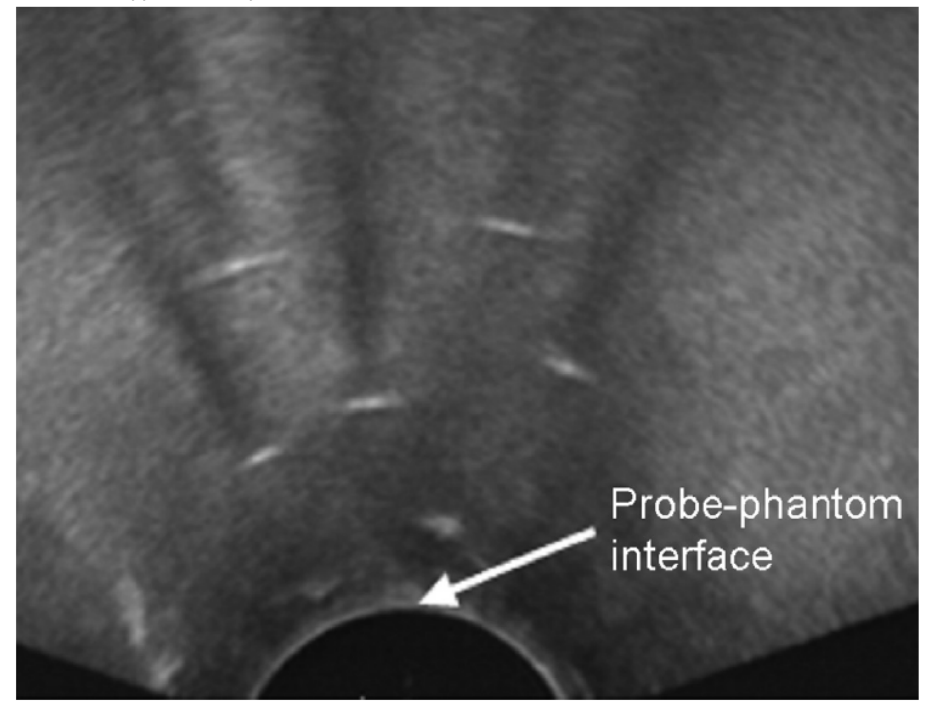

Fig 4. Ultrasound image of the gelatin phantom with the six implanted needles recognizable on the image. The probe-phantom interface is indicated in the image.

During irradiation (total time: 232.1s), the plastic needles were connected to the BT afterloader device through transfer tubes and MOSkins were connected to the CMRP dosimetry system for on- 
line dose measurements. In accordance with the clinical procedure, during irradiation, the TRUS probe was left inside the phantom and its position was reordered in order to identify the position of the detectors. Three measurements in the same irradiation set-up were performed. The resulting measured doses were averaged and compared to the predicted doses in the detector positions on the plan.

\section{Results and Discussion}

\subsection{MOSkin calibration with the Ir-192 brachytherapy source}

The resulting calibration factors for the two employed dosimeters were $0.41 \pm 0.01 \mathrm{cGy} / \mathrm{mV}$ and 0.40 $\pm 0.01 \mathrm{cGy} / \mathrm{mV}$. The corresponding detector sensitivities were $2.43 \mathrm{mV} / \mathrm{cGy}$ and of $2.49 \mathrm{mV} / \mathrm{cGy}$, respectively. Detector sensitivities were similar to those of the MOSkin detectors from other batches which were $2.17 \mathrm{mV} / \mathrm{cG}$ as reported by Qi et al. (2012) and of $2.63 \mathrm{mV} / \mathrm{cGy}$ as reported in Gambarini et al. (2014). Differences between these values might be due to intrinsic differences of the structural and packaging materials between each single dosimeter and to a small sensitivity reduction that might be observed over the entire life time of the detectors.

Concerning the calibration procedure, it is important to note that the reference dose data to perform dosimeter calibration were those resulting from the TPS and cross-checked with software based on the AAPM TG-43 algorithm, rather than those obtained experimentally by the use of a further dosimeter. The choice was taken in accordance to many other studies reported in literature, such as Lambert et al. (2006), Therriault-Proulx et al. (2011) and Gambarini et al. (2014). In fact, factors implemented in the TPS are those tabulated in Daskalov et al. (1998) and are obtained by means of Monte Carlo photon transport code knowing the characteristics of the specific source model (i.e., mHDR-v2). Consensus on these factors has been widely accepted and the resulting TPS calculations are highly accurate when performed in the reference conditions of unbounded water/water-equivalent medium.

\subsection{In phantom dosimetry of prostate brachytherapy treatments}

Table 1. Percentage dose differences between total doses measured by two MOSkin detectors (MOSkin1 and MOSkin2) integrated to the TRUS probe and the expected doses in the same points calculated with the treatment planning system.

\begin{tabular}{llll}
\hline & & & \\
\cline { 2 - 4 } & Dose difference (\%) & Measure\#2 & Measure\#3 \\
\hline MOSkin 1 & 0.05 & 1.3 & 2.8 \\
MOSkin 2 & -4.5 & -2.1 & -1.4 \\
\hline
\end{tabular}

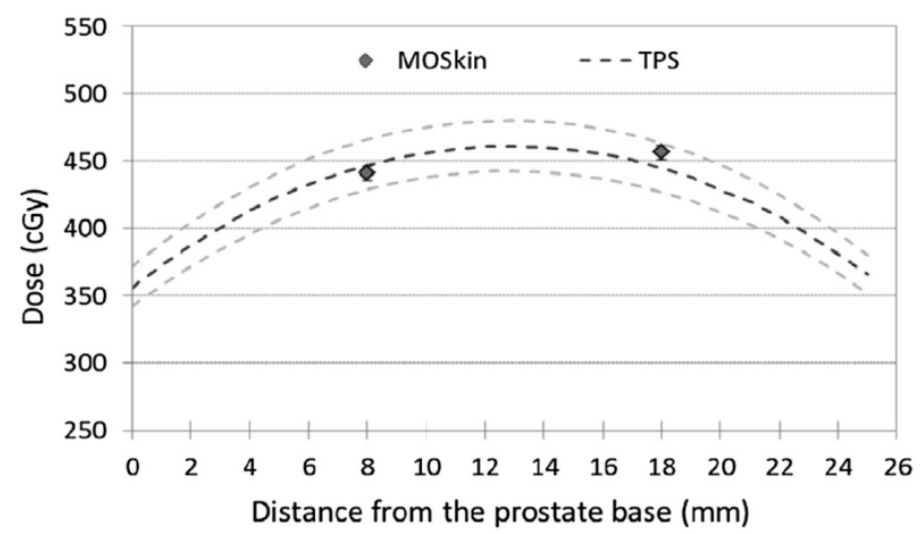

Fig 5. Dose distribution along the longitudinal direction calculated by means of the treatment planning system (TPS) and doses measured by the two MOSkins. The light gray dotted lines indicate the dose distributions calculated $0.5 \mathrm{~mm}$ above and under the probe-phantom interface, which was chosen as the reference radial position of the MOSkins. 
A comparison of total doses measured by MOSkin detectors integrated to the US-probe and the expected doses in the same points calculated with the TPS are reported in Table 1 for the three performed treatment sessions of the same plan. Excellent agreement between values has been observed with a resulting average discrepancy of $0.6 \pm 2.6 \%$. Fig. 5 shows the calculated dose distribution in longitudinal direction and the corresponding average doses measured by the detectors. Inaccuracy in the identification of the exact dosimeter location was of $0.5 \mathrm{~mm}$. Once MOSkins were calibrated in a reference condition, a more complex arrangement representing a typical prostate implant was used to investigate the MOSkin detectors in a clinical setup. In fact, with an accurate and reproducible geometrical disposition of the needles, the study demonstrated that the MOSkins reproducibility was very high and that possible angular and energy dependences (Cygler et al., 2006; Zilio et al., 2006; Hardcastle et al., 2010; Qi et al., 2012) do not significantly affect dose measurements. The obtained results are very promising and of particular clinical importance. Rather than investigating accuracy of the TPS dose calculation, it is expected that in vivo clinical application of MOSkin dosimeters to HDR prostate BT treatments will be very important to discover dose discrepancies due to body movement/anatomy alteration or needle displacement, which may take place between pre-planning imaging and treatment (Milickovic et al., 2011;Dinkla et al., 2013;Cherpak et al.,2014). As this was the first instance of real time dose measurements being performed by integrating dosimeters to the TRUS-probe, the proposed method of dosimetry will be refined through further investigation.

\section{Conclusions}

Real-time dosimetry represents an effective tool for avoiding gross inaccuracies during HDR prostate BT applications. To this aim, MOSkin detectors were integrated to a TRUS probe to perform real time rectal wall in vivo dosimetry without the need to change instrumentation from the devices used for imaging and planning. In phantom results have shown excellent agreement between measurements and calculated data. These results are very promising, however, further in vivo investigation is required to refine the proposed method.

\section{Acknowledgements}

This work was partially supported by the National Institute of Nuclear Physics (INFN), Italy, and by the " 5 per mille" project of the Italian Government.

\section{References}

Batchelar, D., Gaztañaga, M., Schmid, M., Araujo, C., Bachand, F., Crook, J., 2014. Validation study of ultrasound-based high-dose-rate prostate brachytherapy planning compared with CT-based planning. Brachytherapy 13, 75-79.

Cherpak, A.J., Cygler, J.E., Perry, G., 2014. Real-time measurement of urethral dose and position during permanent seed implantation for prostate brachytherapy. Brachytherapy 13, 169-177.

Cygler, J.E., Saoudi, A., Perry, G., Morash, C., Choan, E., 2006. Feasibility study of using MOSFET detectors for in vivo dosimetry during permanent low-dose-rate prostate implants. Radiother. Oncol. 80, 296-301.

Daskalov, G.M., Löffler, E., Williamson, J.F., 1998. Monte Carlo-aided dosimetry of a new high dose-rate brachytherapy source. Med. Phys. 25, 2200-2208.

Dinkla, A.M., Pieters, B.R., Koedooder, K., Meijnen, P., van Wieringen, N., van der Laarse, R., van der Grient, J.N., Rasch, C.R., Bel, A., 2013. Deviations from the planned dose during 48 hours of stepping source prostate brachytherapy caused by anatomical variations. Radiother. Oncol. 107, 106-111.

Fagerstrom, J.M., Micka, J.A., DeWerd, L.A., 2008. Response of an implantable MOSFET dosimeter to 192-Ir HDR radiation. Med. Phys. 35, 5729-5737.

Gambarini, G., Carrara, M., Tenconi, C., Mantaut, N., Borroni, M., Cutajar, D., Petasecca, M., Fuduli, I., Lerch, M., Pignoli, E., Rosenfeld, A., 2014. Online in vivo dosimetry in high dose rate brachytherapy with MOSkin detectors: in phantom feasibility study. Appl. Radiat. Isot. 83, 222-226.

Hardcastle, N., Cutajar, D., Metcalfe, P.E., Lerch, M., Perevertaylo, V.L., Tomé, W.A., Rosenfeld, A.B., 2010. In vivo real-time rectal wall dosimetry for prostate radiotherapy. Phys. Med. Biol. 55, 3859-3871.

Kwan, I.S., Wilkinson, D., Cutajar, D., Lerch, M., Wong, Y., Bucci, J., Perevertaylo, V., Rosenfeld, A.B., 2009. The effect of rectal heterogeneity on wall dose in high dose-rate brachytherapy. Med. Phys. 36, 224-232. 
Lambert, J., McKanzie, D.R., Law, S., Elsey, J., Suchowerska, N., 2006. A plastic scintillation dosimeter for high dose rate brachytherapy. Phys. Med. Biol. 51, 5505-5516.

Lambert, J., Nakano, T., Law, S., Elsey, J., McKenzie, D.R., Suchowerska, N., 2007. In vivo dosimeters for HDR brachytherapy: a comparison of a diamond detector, MOSFET, TLD, and scintillation detector. Med. Phys. 34, 1759-1765.

Milickovic, N., Mavroidis, P., Tselis, N., Nikolova, I., Katsilieri, Z., Kefala, V., Zamboglou, N., Baltas, D., 2011. 4D analysis of influence of patient movement and anatomy alteration on the quality of 3D U/S- based prostate HDR brachytherapy treatment delivery. Med. Phys. 38, 4982-4993.

Morton, G.C., Hopkin, P.J., 2013. Brachytherapy: current status and future strategies - can high dose rate replace low dose rate and external beam radiotherapy? Clin. Oncol. 25, 474-482.

Palmer, A., Bradley, D., Nisbet, A., 2012. Physics-aspects of dose accuracy in high dose rate (HDR) brachytherapy: source dosimetry, treatment planning, equipment performance and in vivo verification techniques. J. Contemp. Brachytherapy 4, 81-91.

Qi, Z.Y., Deng, X.W., Huang, S.M., Lu, J., Lerch, M., Cutajar, D., Rosenfeld, A., 2007. Verification of the plan dosimetry for high dose rate brachytherapy using metaloxide-semiconductor field effect transistor detectors. Med. Phys. 34, 2007-2013.

Qi, Z.Y., Deng, X.W., Cao, X.P., Huang, S.M., Lerch, M., Rosenfeld, A., 2012. A real-time in vivo dosimetric verification method for high dose rate intracavitary brachytherapy of nasopharyngeal carcinoma. Med. Phys. 39, 6756-6763.

Rivard, M.J., Coursey, B.M., DeWerd, L.A., Hanson, W.F., Huq, M.S., Ibbott, G.S., Mitch, M.G., Nath, R., Williamson, J.F., 2004. Update of AAPM task group no. 43 report: a revised AAPM protocol for brachytherapy dose calculations. Med. Phys. 31, 633-674.

Rustgi, S.N., 1998. Application of a diamond detector to brachytherapy dosimetry. Med. Phys. 43, 2085-2094.

Seymour, E.L., Downes, S.J., Fogarty, G.B., Izard, M.A., Metcalfe, P., 2011. In vivo realtime dosimetric verification in high dose rate prostate brachytherapy. Med. Phys. 38, 4785-4794.

Suchowerska, N., Jackson, M., Lambert, J., Yin, Y.B., Hruby, G., McKenzie, D.R., 2011. Clinical trials of a urethral dose measurement system in brachytherapy using scintillation detectors. Int. J. Radiat. Oncol. Biol. Phys. 79, 609-615.

Tanderup, K., Beddar, S., Andersen, C.E., Kertzscher, G., Cygler, J.E., 2013. In vivo dosimery in brachytherapy. Med. Phys. 40 (7), 1-15.

Therriault-Proulx, F., Beddar, S., Briere, T.B., Archambault, L., Beaulieu, L., 2011.

Technical note: removing the stem effect when performing Ir-192 HDR brachytherapy in vivo dosimetry using plastic scintillation detectors: a relevant and necessary step. Med. Phys. 38, 2176-2179.

Thomadsen, B., Lin, S.W., Laemmrich, P.,Waller, T., Cheng, A., Caldwell, B., Rankin, R., Stitt, J., 2003. Analysis of treatment delivery errors in brachytherapy using formal risk analysis techniques. Int. J. Radiat. Oncol. Biol. Phys. 57, 1492-1508.

Toye, W., Das, R., Kron, T., Franich, R., Johnston, P., Duchesne, G., 2009. An in vivo investigative protocol for HDR prostate brachytherapy using urethral and rectal thermoluminescence dosimetry. Radioter. Oncol. 91, 243-248.

Waldhäusl, C., Wambersie, A., Pötter, R., Georg, D., 2005. In-vivo dosimetry for gynaecological brachytherapy: physical and clinical considerations. Radioter. Oncol. 773, 310-317.

Zilio, V., Joneja, O., Popowski, Y., Rosenfeld, A., Chawla, R., 2006. Absolute depth dose-rate measurements for an $192 \mathrm{Ir}$ HDR brachytherapy source in water using MOSFET detectors. Med. Phys. 33, 1532-1539. 